\title{
Efeitos de histórias comportamentais sobre o comportamento de seguir regras discrepantes das contingências ${ }^{1}$
}

\author{
Laercio de Sousa Silva \\ Luiz Carlos de Albuquerque \\ Universidade Federal do Pará
}

\begin{abstract}
RESUMO
Com o objetivo de investigar o papel de variáveis que podem interferir no seguir regras, 10 universitários foram expostos a um procedimento de escolha de acordo com o modelo; a tarefa era apontar cada um dos três estímulos de comparação em uma dada seqüência. Nas duas condições experimentais, nenhuma resposta era reforçada na Sessão 1 (linha de base). As contingências na Sessão 2 eram alteradas na Sessão 3, e as contingências na Sessão 3 eram mantidas inalteradas na Sessão 4, iniciada com a regra discrepante das contingências. A duas condições diferiam apenas na Sessão 2. Na Sessão 2, a seqüência correta era estabelecida por contingências na Condição RD e por regra na Condição IN. Na Condição RD, 4 dos 5 participantes responderam corretamente, de acordo com as contingências, na Sessão 3 e deixaram de seguir a regra discrepante na Sessão 4. Na Condição IN, 4 dos 5 participantes responderam incorretamente na Sessão 3 e seguiram a regra discrepante na Sessão 4. Esses resultados apóiam a sugestão de que a manutenção do seguimento de regra discrepante pode depender das fontes de controle do comportamento alternativo ao especificado por essa regra na história do ouvinte. Discute-se também o papel de outras variáveis.
\end{abstract}

Palavras-chave: regras e contingências; histórias comportamentais; insensibilidade às contingências programadas.

\begin{abstract}
The effects of behavioral history on contingency-discrepant rule-following behavior

With the purpose of investigating the role of variables that may interfere with rule-following, 10 university students were exposed to a matching-to-sample procedure, where they had to point, in sequence, to three comparison stimuli. In the two experimental conditions, neither response was reinforced in Session 1 (baseline). The contingencies in Session 2 were changed in Session 3, and the contingencies in Session 3 remained unchanged in Session 4, which began with a rule that was discrepant to the programmed contingencies. The two conditions differed only in Session 2. In Session 2, the sequence was established by contingencies in Condition RD and by rule in Condition IN. In Condition RD, four of the five participants gave correct responses in accordance with the contingencies in Session 3 and abandoned discrepant rule following in Session 4. In Condition IN, four of the five participants gave incorrect responses in Session 3 and followed the discrepant rule in Session 4. These results suggest that the maintenance of discrepant rule following may depend on the control of behaviors different from those specified by rules in the participant's previous behavioral history. The potential contribution of other variables is also discussed.
\end{abstract}

Keywords: rules and contingencies; behavioral history; insensibility to programmed contingencies.

De modo geral, o controle por regras ${ }^{2}$ tem sido investigado de duas maneiras. Na maneira mais tradicional, observa-se se o comportamento previamente especificado pela regra muda quando as contingências de reforço programadas mudam (por exemplo, Cerutti, 1991; Galizio, 1979; Hayes, Brownstein, Zettle, Rosenfarb \& Korn, 1986; LeFrancois, Chase \& Joyce, 1988; Michael \& Bernstein, 1991; Otto, Torgrud \& Holborn, 1999; Paracampo, Souza, Matos \& Albuquerque, 2001; Shimoff, Catania \& Matthews,
1981). Na outra maneira, observa-se se o comportamento, exposto às contingências de reforço programadas, muda quando as regras mudam (por exemplo, Albuquerque, Reis \& Paracampo, 2006; Albuquerque, Santos, Silva, Mendonça, Queiroz \& Silva; 1993; Albuquerque, de Souza, Matos \& Paracampo, 2003; Martinez \& Tomayo, 2005). Na maneira mais tradicional, a regra é mantida inalterada enquanto que as contingências de reforço programadas no experimento são manipuladas. Na maneira alternativa, as contingências 
de reforço programadas no experimento são mantidas inalteradas, enquanto as regras são manipuladas (Albuquerque e cols., 2003).

Os estudos que têm utilizado o procedimento mais tradicional se originaram, possivelmente, a partir da proposição de Skinner (1969) que sugere que, quando as contingências mudam, e não as regras, o comportamento estabelecido por regras pode não mudar, no sentido de acompanhar as mudanças nas contingências. Neste caso, de acordo com Skinner, as regras poderiam mais atrapalhar do que ajudar. Diferentemente, os estudos que têm utilizado o procedimento alternativo têm procurado testar experimentalmente a proposição de Skinner (1974) que sugere que regras são seguidas porque o comportamento de seguir regras similares foi reforçado no passado. Tais estudos têm investigado os efeitos de histórias de reforço para o seguir regras sobre o seguimento subseqüente de outras regras, quando as contingências de reforço programadas para todas as fases do experimento são mantidas inalteradas (Albuquerque e cols., 2003).

Alguns estudos que têm utilizado o procedimento mais tradicional têm sugerido que é mais provável que o comportamento mude acompanhando as mudanças nas contingências quando é estabelecido por reforço diferencial ou por modelagem do que quando é estabelecido por regras (Paracampo e cols., 2001; Shimoff e cols., 1981). Por exemplo, Paracampo e cols. (2001) expuseram crianças a uma tarefa de discriminação condicional. As crianças da Condição Instrução foram expostas a instruções prévias que descreviam o comportamento que produzia reforço em esquema de reforço contínuo (CRF); já as crianças da Condição Reforço Diferencial não foram expostas a tais instruções. Cinco das oito crianças da Condição Reforço Diferencial aprenderam a executar a tarefa de discriminação condicional e todas as seis crianças da Condição Instrução passaram imediatamente a executar essa tarefa. Quando as contingências mudaram, o comportamento de todas as cinco crianças da Condição Reforço Diferencial mudou acompanhando as mudanças nas contingências, enquanto que o comportamento das seis crianças da Condição Instrução permaneceu inalterado após a mudança nas contingências.

Os estudos que têm utilizado o procedimento alternativo também têm mostrado que o controle por regras pode superar o controle por contingências. Por exemplo, Albuquerque e cols. (2003), expuseram quatro universitários a um procedimento de escolha de acordo com o modelo. A tarefa era apontar para os estímulos de comparação, de acordo com o modelo, em seqüência. Os participantes foram expostos às instruções mínima, discrepante, correspondente e discrepante, nas Fases 1 (linha de base), 2, 3 e 4, respectivamente. Na Fase 1, nenhuma resposta era reforçada. Nas Fases 2 e 4, o seguimento da instrução discrepante não era reforçado. Na Fase 3, o seguimento da instrução correspondente era reforçado em esquema de razão fixa (FR) 4. Todos os quatro participantes apresentaram um desempenho variável na fase de linha de base e todos seguiram tanto a instrução correspondente quanto a instrução discrepante.

Os resultados destes estudos (Albuquerque e cols., 2003; Paracampo e cols., 2001) mostraram que o controle por regras pode superar o controle por contingências. Mas também há evidências mostrando que o controle por contingências pode superar o controle por regras. Por exemplo, Albuquerque e Silva (2006), utilizando um procedimento que combina algumas das características daqueles dois procedimentos (o tradicional e o alternativo) em análise, investigaram os efeitos de uma história de exposição a mudanças nas contingências sobre o seguimento subseqüente de regras discrepantes das contingências. Neste estudo, nove universitários foram expostos a um procedimento de escolha de acordo o modelo. Em cada tentativa, um estímulo modelo e três de comparação eram apresentados ao participante e, em seguida, uma de duas lâmpadas (estímulos contextuais) era acesa. Na presença desses estímulos, o participante deveria apontar para os três estímulos de comparação em uma dada seqüência. As seqüências corretas eram reforçadas em CRF. O experimento era constituído de três condições e cada condição de quatro sessões. As contingências na Sessão 1 eram alteradas na Sessão 2, restabelecidas na Sessão 3 e mantidas inalteradas na Sessão 4, que era iniciada com a apresentação da instrução discrepante. No início da Sessão 1, os participantes da Condição 1 foram expostos à instrução mínima, os da Condição 2, à instrução correspondente para o comportamento não-verbal, e os da Condição 3, à instrução correspondente para o comportamento verbal. Todos os participantes, durante cada condição, foram solicitados a verbalizar e as suas verbalizações corretas eram reforçadas.

Sete participantes [três da Condição 1, dois da Condição 2 e dois da Condição 3 (um desses dois participantes da Condição 3 não seguiu a instrução na Sessão 1 e, portanto, teve seu comportamento estabelecido por reforço diferencial nessa sessão)] apresentaram desempenhos não-verbal e verbal sob controle 
das contingências nas Sessões 2 e 3 e deixaram de seguir a regra discrepante na Sessão 4; e dois (um da Condição 2 e um da Condição 3) apresentaram desempenhos não-verbal e verbal independentes das conseqüências programadas nas Sessões 2 e 3 (isto é, continuaram seguindo a instrução correspondente) e seguiram a instrução discrepante na Sessão 4.

Os autores propuseram que o comportamento de seguir regras discrepantes das contingências pode ser mantido, ou não, dependendo das fontes de controle do comportamento alternativo ao especificado pela regra discrepante na história do ouvinte. Ou seja, propuseram que o seguimento de regra discrepante das contingências: 1 ) tende a ser mantido quando, antes da apresentação da regra, o comportamento alternativo ao especificado pela regra discrepante não se mostra sob controle das conseqüências programadas; e 2) tende a deixar de ocorrer quando, antes da apresentação da regra, este comportamento alternativo mostra-se sob controle das conseqüências programadas. Propuseram também que isto pode ocorrer independentemente de se, antes das mudanças nas contingências de reforço, o comportamento é inicialmente estabelecido por regras ou por reforço diferencial, uma vez que dos sete participantes que deixaram de seguir a regra discrepante na Sessão 4, três tiveram o comportamento estabelecido por regra correspondente e quatro tiveram o comportamento estabelecido por reforço diferencial na Sessão 1.

Contudo, não fica claro por que, diferente do que tem sido proposto na literatura (Paracampo e cols., 2001; Shimoff e cols., 1981), três dos cinco participantes, que tiveram o comportamento estabelecido por regra correspondente, no estudo de Albuquerque e Silva (2006), abandonaram o seguimento de regra quando as contingências mudaram. É possível que essas diferenças de resultados tenham ocorrido devido a diferenças nas histórias pré-experimentais dos participantes (Branch, 1991). Mas também é possível supor que a maior parte dos participantes deste estudo, que tiveram o comportamento estabelecido por regra correspondente, tenha abandonado o seguimento de regra, após a mudança nas contingências, porque neste estudo havia condições que favoreciam o controle pelas contingências programadas.

Por exemplo, pode-se supor que o comportamento estabelecido por regra mudou acompanhando a mudança nas contingências neste estudo, porque a tarefa exigida dos participantes era complexa. Ou seja, a tarefa dos participantes consistia em emitir duas seqüências com três respostas cada uma [por exemplo, o participante deveria responder na seqüência forma espessura - cor (isto é, deveria apontar primeiro para o estímulo de comparação que tivesse a mesma forma, depois para o que tivesse a mesma espessura e, em seguida, para o que tivesse a mesma cor do estímulo modelo) quando uma lâmpada estivesse acesa; e responder na seqüência espessura-forma-cor quando uma outra lâmpada estivesse acesa]. Pode-se supor também que os participantes deste estudo apresentaram um desempenho sob controle de suas conseqüências, quando as contingências mudaram, porque as perguntas acerca de qual comportamento produzia reforço, feitas ao longo das sessões, facilitaram a discriminação da discrepância entre a regra e as conseqüências produzidas pelo seguimento de regra. Além disso, a discriminação dessa discrepância também pode ter sido facilitada, porque neste estudo o comportamento era reforçado em esquema CRF, antes e depois da mudança nas contingências.

Se, como essas suposições sugerem, a manipulação desses fatores (a complexidade da tarefa, as verbalizações dos participantes e o tipo de esquema de reforço) pode interferir no seguimento de regras, deveria ser esperado que o seguimento da regra correspondente fosse mantido, após a mudança nas contingências, caso fosse utilizado um procedimento similar ao usado por Albuquerque e Silva (2006), mas, diferente deste estudo, fosse usada uma tarefa experimental mais simples, o comportamento verbal dos participantes não fosse solicitado e fossem utilizados um esquema de reforço intermitente (antes da mudança nas contingências) e um esquema de reforço contínuo (depois dessa mudança). Se for assim, também deveria ser esperado que o seguimento subseqüente da regra discrepante fosse mantido, uma vez que este comportamento tende a persistir quando, antes da apresentação dessa regra, o comportamento alternativo ao por ela especificado não se mostra sob controle das conseqüências programadas (Albuquerque \& Silva, 2006). O presente estudo procurou avaliar essas possibilidades.

Além disso, também procurou avaliar os eventuais efeitos daquelas alterações no procedimento usado por Albuquerque e Silva (2006) sobre a sensibilidade ${ }^{3}$ do comportamento estabelecido por reforço diferencial à mudança nas contingências. Neste caso, deveria ser esperado que os participantes mudassem o comportamento, quando as contingências fossem alteradas, uma vez que uma característica do comportamento estabelecido por contingências é que ele tende a ser sensível às suas conseqüências (Chase \& Danforth, 1991; 
Shimoff e cols., 1981). Se for assim, deveria ser esperado que o seguimento subseqüente da regra discrepante fosse abandonado, uma vez que este comportamento tende a deixar de ocorrer quando, antes da apresentação dessa regra, o comportamento alternativo ao por ela especificado mostra-se sob controle das conseqüências programadas (Albuquerque e Silva, 2006).

Assim, o presente estudo procurou construir duas histórias experimentais. Em uma, procurou-se primeiro estabelecer o comportamento por reforço diferencial e, depois, testar a sensibilidade desse comportamento à mudança nas contingências programadas. Em outra, procurou-se primeiro estabelecer o comportamento por regra correspondente às contingências de reforço e, depois, testar a sensibilidade desse comportamento à mudança nas contingências programadas. $\mathrm{O}$ presente estudo procurou fazer isso com o objetivo de também avaliar os efeitos de cada uma dessas duas histórias sobre o seguimento subseqüente de uma regra discrepante das contingências. Em síntese o presente estudo teve o objetivo de: 1 ) verificar se o comportamento estabelecido ou por reforço diferencial ou por regra correspondente é mantido, ou não, após a mudança nas contingências; e, 2) avaliar os efeitos, sobre o seguimento subseqüente de uma regra discrepante das contingências programadas, de uma história de um comportamento estabelecido por reforço diferencial e exposto à mudança nas contingências e de uma história de um comportamento estabelecido por regra correspondente e exposto à mudança nas contingências, quando: a) a tarefa dos participantes é a de emitir uma seqüência com três respostas; b) os participantes não são solicitados a verbalizar; e, c) o seguimento da regra correspondente é reforçado em esquema FR 2, antes da mudança nas contingências, e em esquema CRF, depois da mudança nas contingências. Para tanto, foi utilizado um procedimento de escolha de acordo com o modelo similar ao usado por Albuquerque e Silva (2006).

No presente estudo, será dito que o comportamento que se seguir à apresentação de uma regra está sob controle de regra, quando a combinação de duas condições for satisfeita: 1) quando o comportamento observado for o especificado na regra, emitido na presença dos estímulos descritos pela regra, e na seqüência descrita na regra; e, 2) esse comportamento ocorrer independentemente das conseqüências programadas no experimento.

Será dito que o comportamento observado está sob controle das contingências de reforço programadas no experimento quando a combinação de duas condições for satisfeita: 1) quando o comportamento observado for o reforçado; e, 2) este comportamento ocorrer independentemente de uma descrição antecedente verbal, especificando que comportamento na presença de que estímulo poderá ser reforçado.

E será dito que o comportamento que se seguir à apresentação de uma regra está sob controle da interação entre a regra e as contingências de reforço, quando a combinação de duas condições for satisfeita: 1) quando o comportamento observado for o especificado na regra, emitido na presença dos estímulos descritos pela regra, e na seqüência descrita na regra; e, 2) este comportamento estiver sendo reforçado.

\section{MÉTODO}

\section{Participantes}

Participaram 10 estudantes universitários, de diversos cursos (exceto o de Psicologia), oito homens e duas mulheres, com idades variando entre 18 e 32 anos e matriculados em diferentes semestres. Todos foram convidados a participar do experimento, como se segue: "Estou realizando uma pesquisa sobre aprendizagem e queria saber se você estaria interessado em participar. $\mathrm{O}$ objetivo da pesquisa é investigar processos de aprendizagem comuns a todas as pessoas e será realizada no laboratório de Psicologia, aqui da universidade. A pesquisa será realizada em um único dia da semana e deve ter a duração de duas horas, aproximadamente. A pessoa que participar receberá a passagem de ônibus de ida e volta à universidade. Além disso, poderá receber mais uma quantia em dinheiro no final da pesquisa. Você está interessado em participar?”

\section{Equipamento e material}

Foi utilizada uma mesa de madeira. Fixado à mesa, de modo a dividi-la ao meio, havia um anteparo com espelho unidirecional, fixado em uma moldura de madeira e localizado acima do tampo da mesa. No centro do anteparo, junto ao tampo da mesa, havia uma abertura retangular. Acima e ao centro desta abertura, havia um contador operado pelo experimentador e com os dígitos voltados para o participante. Visível ao participante estava instalada no anteparo uma lâmpada transparente de 5 watts com uma etiqueta de papel com a frase impressa: "Você ganhou um ponto”. Uma lâmpada fluorescente de 15 watts estava instalada na borda superior e ao centro do anteparo. 
Os estímulos modelo e de comparação foram peças de madeira (blocos lógicos da marca Funbec), variando em três dimensões: forma (quadrado, círculo, retângulo e triângulo), cor (azul, vermelha e amarela) e espessura (grossa e fina). Estas peças de madeira formavam 30 diferentes arranjos de estímulos, cada um constituído de um estímulo modelo e três estímulos de comparação. Cada estímulo de comparação apresentava apenas uma dimensão - cor (C), espessura (E) ou forma (F) - em comum com o estímulo modelo e diferia nas demais. A combinação dos estímulos era aleatória. Os 30 arranjos de estímulos previamente preparados ficavam sobre a mesa, ao lado do experimentador, na ordem em que seriam apresentados em cada tentativa. Para facilitar o manejo dos arranjos, sentado ao lado esquerdo do experimentador, um auxiliar de pesquisa aproximava os arranjos, conforme os mesmos iam sendo apresentados. Os estímulos eram apresentados ao participante através da abertura retangular na base do anteparo divisor da mesa, em uma bandeja de madeira em forma de ' $T$ '. Na parte final do cabo dessa bandeja, rente à base retangular, quatro ripas de madeira formavam um quadrado, no qual era colocado o estímulo modelo. Na base retangular da bandeja, dividida por ripas de madeira em três quadrados, eram apresentados os três estímulos de comparação. As respostas de escolha emitidas pelos participantes eram registradas pelo experimentador em um protocolo previamente preparado e eram também gravadas por uma filmadora, para análises posteriores. Os reforçadores utilizados eram pontos, registrados no contador. Cada ponto valia R\$ 0,05 (cinco centavos de real).

\section{Procedimento}

Durante as sessões experimentais, participante e experimentador ficavam sentados à mesa de frente um para o outro, separados pelo anteparo divisor da mesa. A lâmpada na borda superior do anteparo ficava constantemente acesa, voltada para o participante, de maneira a assegurar que seu lado apresentasse iluminação em maior intensidade, garantindo que as ações emitidas pelo participante, bem como os arranjos de estímulos apresentados, pudessem ser observados através do espelho. O experimentador, em algumas sessões, inicialmente apresentava ao participante uma determinada instrução e em seguida apresentava os arranjos de estímulos, em outras, apresentava apenas os arranjos de estímulos. Cada sessão durava em média 20 min e o intervalo entre sessões era de aproximadamente 5 min. Em cada tentativa, após o experimentador apresentar um dos 30 arranjos de estímulos, e enquanto este ainda estava presente, o participante deveria apontar para os estímulos de comparação, de acordo com o modelo, em uma dada seqüência. As seqüências corretas eram reforçadas com pontos trocados por dinheiro no final da pesquisa. Os pontos eram registrados cumulativamente, no contador, apenas dentro de uma mesma sessão. No entanto, a partir da segunda sessão, logo após entrar na sala experimental, o participante era informado pelo experimentador sobre o número total de pontos obtidos nas sessões anteriores. Caso a sequiência de respostas emitida estivesse de acordo com as contingências de reforço programadas (seqüência correta), a lâmpada transparente com a frase impressa: "Você ganhou um ponto" era acesa e apagada, um ponto era acrescentado no contador e a bandeja com o arranjo de estímulos era retirada. Caso a seqüência de respostas fosse incorreta, a lâmpada transparente não era acesa e a bandeja com o arranjo de estímulos era retirada, sem ser acrescentado um ponto no contador. Havia um intervalo variável de aproximadamente $5 \mathrm{~s}$ entre uma tentativa e outra.

\section{Orientações preliminares}

Na primeira sessão, quando participante e experimentador entravam na sala, a bandeja com um arranjo de estímulos estava sobre a mesa, portanto, visível ao participante. O experimentador pedia ao participante para sentar-se na cadeira e, ao lado do participante, sempre apontando com o dedo para cada um dos estímulos a que se referia, dizia:

Este objeto, aqui em cima, é um modelo. Estes três objetos, aqui em baixo, são para você comparar com o modelo. Nós vamos chamar estes três objetos, aqui em baixo, de objetos de comparação. Observe que cada um destes três objetos de comparação tem uma única propriedade comum ao modelo. [Veja: este só tem a espessura comum ao modelo, este aqui só tem a cor comum ao modelo, este aqui só tem a forma igual ao modelo]. Durante a pesquisa você poderá ganhar pontos que serão trocados por dinheiro. Quando você ganhar pontos, os pontos sempre aparecerão aqui neste contador e esta lâmpada será acesa. Cada ponto que você ganhar será trocado por $R \$ 0,05$ (cinco centavos de real), mas apenas no final da pesquisa. Veja como os pontos aparecem no contador (o auxiliar, que se encontrava no outro lado da mesa, acionava o contador por cinco vezes). Quando você não ganhar pontos, nenhum ponto será acrescentado no contador e esta lâmpada não será acesa. Entendeu?

Este procedimento era repetido duas vezes e ocorria apenas no início da primeira sessão. Na segunda 
vez em que essas orientações preliminares eram apresentadas, o trecho entre colchetes era omitido.

\section{Instruções}

A seguir, o experimentador pedia para o participante colocar os fones de ouvido e se deslocava em direção à sua cadeira. Separado do participante pelo anteparo com espelho unidirecional, o experimentador também colocava os seus fones de ouvido e, dependendo da sessão experimental, entregava ao participante, pela abertura na base do anteparo, uma folha de papel contendo uma das seguintes instruções (regras) impressas:

- Instrução mínima: Instrução que não especificava seqüência de respostas.

Aponte com o dedo em seqüência para cada um dos três objetos de comparação.

- Instrução correspondente: Instrução cujo comportamento de segui-la era reforçado.

Quando eu mostrar estes objetos para você, você deve fazer o seguinte: Primeiro aponte com o dedo para o objeto de comparação que tem a mesma cor [espessu$\left.\mathrm{ra}^{4}\right]$ do objeto modelo. Depois aponte para o objeto de comparação que tem a mesma espessura [cor] do objeto modelo. Em seguida aponte para o objeto de comparação que tem a mesma forma [forma] do objeto modelo. Ou seja, você deve apontar primeiro para a mesma cor [espessura], depois para a mesma espessura [cor] e, em seguida, para a mesma forma [forma]. Entendeu? Repita para mim o que você deve fazer. Fazendo isso, você poderá ganhar pontos, que serão mostrados no contador à sua frente. Cada ponto que você ganhar será trocado por R\$ 0,05 (cinco centavos de real), mas apenas no final da pesquisa.

- Instrução discrepante: Instrução cujo comportamento de segui-la não era reforçado.

Quando eu mostrar estes objetos para você, você deve fazer o seguinte: Primeiro aponte com o dedo para o objeto de comparação que tem a mesma forma do objeto modelo. Depois aponte para o objeto de comparação que tem a mesma cor do objeto modelo. Em seguida aponte para o objeto de comparação que tem a mesma espessura do objeto modelo. Ou seja, você deve apontar primeiro para a mesma forma, depois para a mesma cor e, em seguida, para a mesma espessura. Entendeu? Repita para mim o que você deve fazer. Fazendo isso, você poderá ganhar pontos que serão mostrados no contador à sua frente. Cada ponto que você ganhar será trocado por $R \$ 0,05$ (cinco centavos de real), mas apenas no final da pesquisa.

No início das Sessões 1 e 4 da Condição Reforço Diferencial (descrita a seguir) e no início das Sessões 1, 2 e 4 da Condição Instrução (também descrita a seguir), imediatamente após entregar ao participante a folha de papel contendo uma instrução impressa, o experimentador ligava o tape-deck e, por intermédio dos fones de ouvido, o participante passava a ouvir uma fita, previamente gravada, com a voz do experimentador lendo a instrução. Na gravação, o experimentador solicitava ao participante, ora que acompanhasse a sua leitura, ora que ele lesse sozinho, silenciosamente. Desse modo, o participante lia a instrução por três vezes seguidas no início de cada uma destas sessões. Após a última leitura, a gravação solicitava ao participante que devolvesse a folha com a instrução. Logo após receber a folha com a instrução, o experimentador removia a bandeja, voltava a apresentar a bandeja com um novo arranjo de estímulos, e dizia: "Comece a apontar".

Os participantes foram distribuídos em duas condições experimentais. Cada condição, realizada com cinco participantes, era constituída de quatro sessões. A duas condições diferiam apenas quanto à forma de estabelecimento do comportamento na Sessão 2. Na Sessão 2 da Condição RD (Reforço Diferencial), o comportamento era estabelecido por reforço diferencial, enquanto na Sessão 2 da Condição IN (Instrução), o comportamento era estabelecido por instrução correspondente às contingências de reforço programadas nesta sessão.

\section{Condição RD}

A Sessão 1 da Condição RD era iniciada com a apresentação da instrução mínima, as Sessões 2 e 3 eram iniciadas com a apresentação dos arranjos de estímulos, uma vez que nestas sessões não eram apresentadas instruções, e a Sessão 4 com a instrução discrepante das contingências de reforço.

Durante a Sessão 1 eram apresentados apenas 10 dos 30 diferentes arranjos de estímulos e nenhuma resposta era reforçada. Ou seja, a Sessão 1 era constituída de 10 tentativas de linha de base em relação à qual eram avaliados os efeitos da introdução do procedimento de reforço diferencial na Sessão 2.

Durante a Sessão 2, apenas uma de duas seqüências de respostas era reforçada com pontos trocáveis por dinheiro: a emissão da seqüência CEF [em outras 
palavras, o apontar primeiro para o estímulo de comparação que tivesse a mesma cor (C), depois para o que tivesse a mesma espessura (E) e, em seguida, para o que tivesse a mesma forma (F) do estímulo modelo] ou a emissão da seqüência ECF. Qual dessas seqüências seria reforçada e, portanto, considerada correta, dependia do desempenho do participante na Sessão 1. Se na Sessão 1 a seqüência CEF fosse emitida em mais de $50 \%$ das tentativas, a seqüência correta na Sessão 2 seria a seqüência alternativa $\mathrm{ECF}$, e não a seqüência CEF. Caso contrário, a seqüência correta na Sessão 2 seria a seqüência $\mathrm{CEF}$, e não a seqüência alternativa ECF. Esse procedimento foi usado para evitar coincidência entre o comportamento emitido pelo participante em uma determinada sessão e o seu comportamento apresentado na sessão subseqüente. No início da Sessão 2, a seqüência correta (CEF ou ECF), era reforçada em CRF. Imediatamente após o participante receber 10 pontos consecutivos em CRF, a seqüência correta passava a ser reforçada em esquema FR 2. Nesse esquema de razão fixa, cada duas emissões consecutivas da seqüência correta produzia um ponto no contador. Erros, ou a não-emissão consecutiva da seqüência correta, reiniciavam o esquema FR 2 para obtenção de um ponto. A Sessão 2 era encerrada após a obtenção de 10 pontos em FR 2, independentemente de serem consecutivos ou não. Caso, na Sessão 2, o participante não ganhasse pontos em 30 tentativas consecutivas, a sua participação no experimento era encerrada nesta sessão. Portanto, só eram expostos às Sessões 3 e 4, os participantes que atingissem o critério de desempenho para o encerramento da Sessão 2, isto é, os que ganhassem 10 pontos em FR 2. A transição da Sessão 2 para a Sessão 3 era marcada pela mudança, não sinalizada, nas contingências de reforço programadas.

Durante a Sessão 3, apenas a emissão da seqüência EFC era reforçada. Essa seqüência era reforçada em CRF. Essa sessão era encerrada de acordo com um dos seguintes critérios, o que ocorresse primeiro: após a obtenção de 10 pontos ou após a ocorrência de 30 tentativas. A transição da Sessão 3 para a Sessão 4 era marcada pela introdução da instrução discrepante no início da Sessão 4.

Durante a Sessão 4, a emissão da seqüência EFC continuava sendo reforçada em CRF. Esta era a única seqüência reforçada na Sessão 4. Essa sessão era encerrada de acordo com um dos seguintes critérios, o que ocorresse primeiro: após a obtenção de 10 pontos ou após a ocorrência de 30 tentativas.

\section{Condição IN}

A Condição IN era idêntica à Condição RD, exceto na Sessão 2. A Sessão 2 da Condição IN foi planejada para ser iniciada com a instrução correspondente especificando a seqüência CEF. Contudo, se na Sessão 1 o participante respondesse na seqüência CEF em mais de $50 \%$ das tentativas, a Sessão 2 seria iniciada com a instrução correspondente especificando a seqüência alternativa ECF, e não a seqüência CEF. A única seqüência reforçada nesta sessão era a seqüência que a instrução correspondente especificasse, fosse CEF ou ECF. A seqüência especificada pela instrução correspondente era reforçada, desde o início da sessão, em esquema FR 2. Esse procedimento foi usado para separar o controle por regras do controle pelas conseqüências programadas nas duas primeiras tentativas da Sessão 2 desta condição. Desse modo, a Sessão 2 da Condição IN era encerrada após a obtenção de 10 pontos em FR 2, independentemente de serem consecutivos ou não.

Nas duas condições, a cada duas sessões, um observador independente comparava o registro feito pelo experimentador com o registro feito pela filmadora. Caso houvesse $100 \%$ de concordância entre os registros, o participante continuava participando do experimento. Caso contrário, o participante seria descartado. No presente estudo, nenhum participante foi descartado. A participação do estudante no experimento era encerrada quando o participante atingisse o critério de encerramento da Sessão 4, ou se o participante não atingisse o critério de encerramento da Sessão 2.

\section{RESULTADOS}

Os dados dos participantes, tanto da Condição RD (Reforço Diferencial) quanto da Condição IN (Instrução), são apresentados nas Tabelas 1 e 2 e na Figura 1. A Tabela 1 mostra as porcentagens de seqüências de respostas emitidas durante a Sessão 1 (linha de base). Já a Tabela 2 mostra os dados dos participantes que atingiram o critério de desempenho para o encerramento da Sessão 2, relativos ao número ordinal da tentativa: a) na qual os participantes emitiram a seqüência correta pela primeira vez nas Sessões 2, 3 e 4; e, b) na qual o critério de desempenho para o encerramento de sessão foi atingido. $\mathrm{E}$ a Figura $1^{5}$ mostra a freqüência acumulada de seqüências de respostas corretas e incorretas emitidas pelos participantes.

Na Tabela 1, observa-se que cinco participantes (P12, P13, P14, P21 e P24) apresentaram um desem- 
penho variável na Sessão 1. Já os Participantes P11, P15, P22, P23 e P25, responderam, em mais de 50\% das tentativas dessa sessão, nas seqüências ECF, ECF, ECF, CFE e FCE, respectivamente. Portanto, como nenhum participante chegou a responder sistematicamente na seqüência CEF na Sessão 1 , a seqüência
CEF passou a ser a seqüência correta (reforçada) na Sessão 2 das duas condições. Desse modo, a Sessão 2 da Condição RD foi iniciada reforçando-se diferencialmente as emissões da seqüência CEF e a Sessão 2 da Condição IN foi iniciada com a instrução correspondente especificando a seqüência CEF.

TABELA 1

Porcentagens de Seqüências de Respostas Emitidas Durante a Sessão 1

\begin{tabular}{ccccccc}
\hline Condição RD (Reforço Diferencial) & \multicolumn{7}{c}{ Seqüências } \\
\cline { 2 - 6 } Participantes & CEF & CFE & FCE & FEC & EFC & ECF \\
\hline P 11 & 20 & 0 & 0 & 10 & 0 & 70 \\
P 12 & 10 & 20 & 20 & 0 & 30 & 20 \\
P 13 & 20 & 0 & 30 & 10 & 20 & 20 \\
P 14 & 20 & 10 & 10 & 30 & 10 & 20 \\
P 15 & 0 & 0 & 0 & 0 & 0 & 100 \\
\hline Condição IN (Instrução) & & & & & & \\
& & & Seqüências & & 10 & \\
Participantes & CEF & CFE & FCE & FEC & EFC & ECF \\
\hline P 21 & 10 & 20 & 20 & 30 & 10 & 10 \\
P 22 & 0 & 0 & 20 & 0 & 10 & 70 \\
P 23 & 10 & 60 & 0 & 0 & 20 & 10 \\
P 24 & 20 & 10 & 30 & 10 & 10 & 20 \\
P 25 & 0 & 0 & 60 & 10 & 30 & 0 \\
\hline
\end{tabular}

Nota. $\mathrm{C}=$ resposta à dimensão cor; $\mathrm{E}=$ resposta à espessura; $\mathrm{F}=$ resposta à forma.

Na Figura 1 e na Tabela 2, pode-se observar que quatro (P11, P12, P13 e P14) dos cinco participantes da Condição RD atingiram o critério de desempenho para o encerramento da Sessão 2 (ou seja, a obtenção de 10 pontos em FR 2), emitindo a seqüência de respostas correta (seqüência CEF). O Participante P15 não atingiu este critério de encerramento da Sessão 2, uma vez que não ganhou pontos em 30 tentativas consecutivas e, portanto, não foi exposto às Sessões 3 e 4. Os Participantes P11, P12, P13 e P14 iniciaram a Sessão 2 respondendo incorretamente. A partir das Tentativas 3, 6, 3 e 5, respectivamente, começaram a responder corretamente e atingiram o critério de desempenho para o encerramento da Sessão 2 nas Tentativas 38, 81, 46 e 36, respectivamente. Na Sessão 3, quando as contingências de reforço foram alteradas, estes quatro participantes mudaram os seus desempenhos e passaram a responder corretamente (isto é, passaram a emitir a seqüência EFC), de acordo com as novas contingências de reforço. Na Sessão 4, quando a instrução discrepante foi introduzida e as contingências em vigor na Sessão 3 foram mantidas inalteradas, todos os quatro participantes iniciaram esta sessão respondendo incorretamente, seguindo a instrução discrepante (isto é, emitindo a seqüência FCE). Mas depois, esses participantes abandonaram o seguimento da instrução e passaram a responder corretamente (isto é, passaram a emitir a seqüência EFC), de acordo com as contingências programadas na Sessão 4.

Também se pode observar, na Figura 1 e na Tabela 2, que os Participantes P21, P22, P23, P24 e P25 da Condição IN iniciaram a Sessão 2 respondendo corretamente, seguindo a instrução correspondente desde a primeira tentativa, e atingiram o critério de desempenho para o encerramento da Sessão 2 nas Tentativas 24, 20, 22, 20 e 20, respectivamente. Na Sessão 3, quando as contingências de reforço foram alteradas e o seguimento da instrução (apresentada no início da 
Sessão 2) deixou de produzir pontos, quatro participantes (P21, P22, P23 e P24) continuaram seguindo a instrução (isto é, continuaram emitindo a seqüência CEF) e um (P25) mudou o seu desempenho, deixando de seguir a instrução e passando a responder corretamente, de acordo com as novas contingências de reforço. Na Sessão 4, quando a instrução discrepante foi introduzida, todos os cinco participantes da Condição IN iniciaram esta sessão respondendo incorretamente, seguindo a instrução discrepante. Depois, os quatro participantes (P21, P22, P23 e P24), que tinham seguido a instrução na Sessão 3, continuaram seguindo a instrução discrepante e o Participante P25, que havia deixado de seguir a instrução na Sessão 3, abandonou o seguimento da instrução discrepante e passou a responder corretamente, de acordo com as contingências de reforço programadas na Sessão 4.
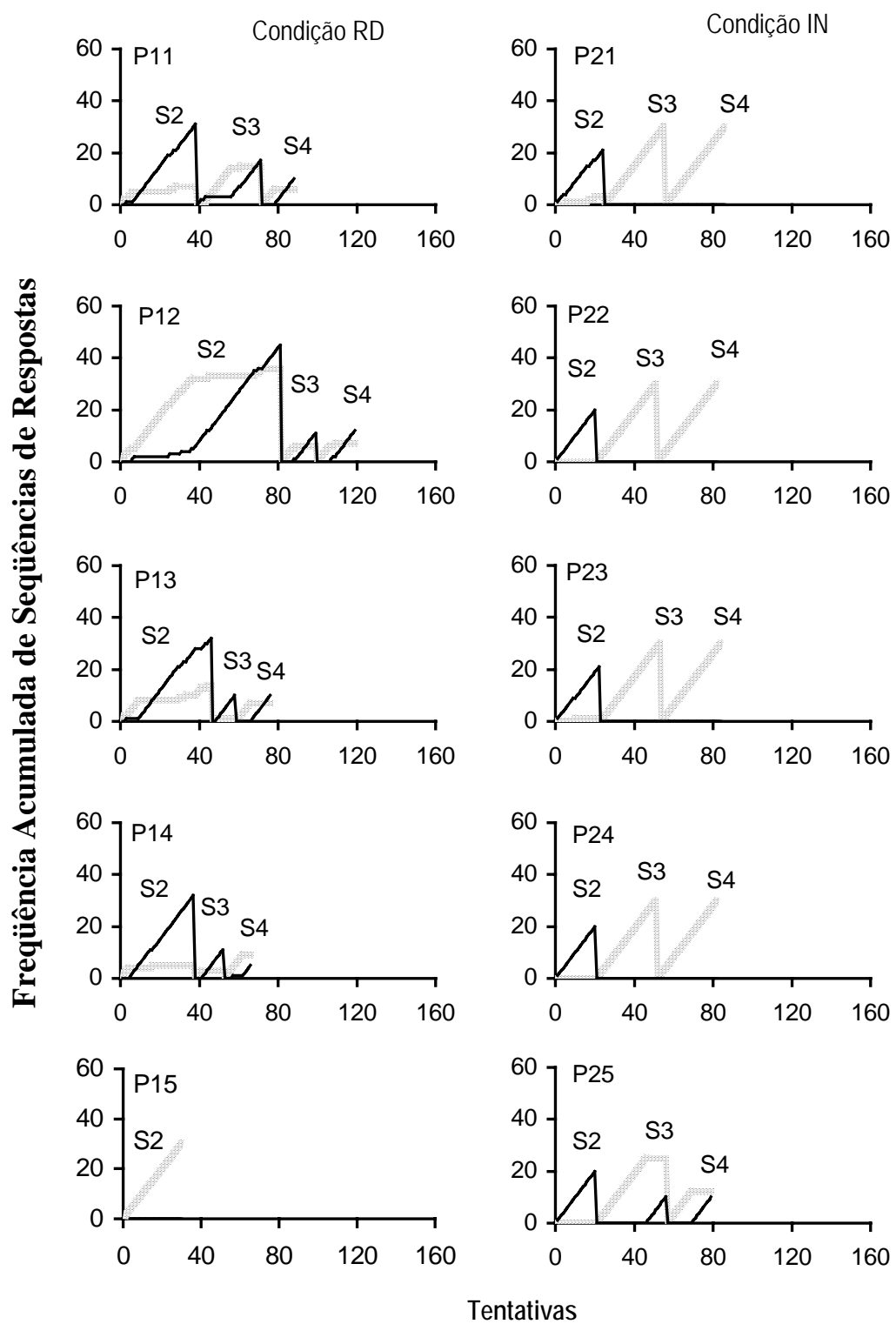

Figura 1. Freqüência acumulada de seqüências de respostas corretas (linha sólida preta) e incorretas (linha pontilhada cinza), para cada participante (P), durante as Sessões (S) 2, 3 e 4. Quebras na curva acumulada indicam mudanças de sessão. 
TABELA 2

Resumo do Desempenho Apresentado Pelos Participantes das Duas Condições

\begin{tabular}{|c|c|c|c|c|c|c|}
\hline \multicolumn{7}{|c|}{ Condição RD (Reforço Diferencial) } \\
\hline \multirow[b]{2}{*}{ Participantes } & \multicolumn{3}{|c|}{$\begin{array}{l}\text { Tentativa na qual a seqüência correta } \\
\text { foi emitida pela primeira vez dentro } \\
\text { de cada sessão }\end{array}$} & \multicolumn{3}{|c|}{$\begin{array}{l}\text { Tenativa na qual o critério de } \\
\text { desempenho para o encerramento } \\
\text { de sessão foi atingido }\end{array}$} \\
\hline & Sessão 2 & Sessão 3 & Sessão 4 & Sessão 2 & Sessão 3 & Sessão 4 \\
\hline P 11 & 3 & 1 & 7 & 38 & 32 & 16 \\
\hline P 12 & 6 & 6 & 7 & 81 & 17 & 19 \\
\hline P 13 & 3 & 2 & 8 & 46 & 11 & 17 \\
\hline P 14 & 5 & 4 & 10 & 36 & 13 & 19 \\
\hline
\end{tabular}

Condição IN (Instrução)

\begin{tabular}{|c|c|c|c|c|c|c|}
\hline \multirow[b]{2}{*}{ Participantes } & \multicolumn{3}{|c|}{$\begin{array}{l}\text { Tentativa na qual a seqüência correta } \\
\text { foi emitida pela primeira vez dentro } \\
\text { de cada sessão }\end{array}$} & \multicolumn{3}{|c|}{$\begin{array}{l}\text { Tenativa na qual o critério de } \\
\text { desempenho para o encerramento } \\
\text { de sessão foi atingido }\end{array}$} \\
\hline & Sessão 2 & Sessão 3 & Sessão 4 & Sessão 2 & Sessão 3 & Sessão 4 \\
\hline P 21 & 1 & Não & Não & 24 & Não & Não \\
\hline P 22 & 1 & Não & Não & 20 & Não & Não \\
\hline P 23 & 1 & Não & Não & 22 & Não & Não \\
\hline P 24 & 1 & Não & Não & 20 & Não & Não \\
\hline P 25 & 1 & 26 & 6 & 20 & 35 & 32 \\
\hline
\end{tabular}

Nota. "Não" indica que o participante não respondeu corretamente na sessão e, portanto, que a sessão foi encerrada pelo número de tentativas, isto é, após a ocorrência de 30 tentativas.

\section{DISCUSSÃO}

Em síntese, os resultados do presente estudo mostraram que, na Condição RD (Reforço Diferencial), quatro (P11, P12, P13 e P14) dos cinco participantes atingiram o critério de desempenho para o encerramento da Sessão 2, mudaram o comportamento quando as contingências de reforço mudaram na Sessão 3 e deixaram de seguir a instrução discrepante das contingências de reforço na Sessão 4. Em contraste, na Condição IN (Instrução), quatro (P21, P22, P23 e P24) dos cinco participantes atingiram o critério de desempenho para o encerramento da Sessão 2, não mudaram o comportamento quando as contingências de reforço mudaram na Sessão 3 (continuaram seguindo a instrução na Sessão 3) e seguiram a instrução discrepante na Sessão 4. Quanto aos dois participantes restantes, P15, da Condição $\mathrm{RD}$, teve sua participação encerrada na Sessão 2, uma vez que não atingiu critério de desempenho para o encerramento dessa sessão. Já P25, da Condição IN, apresentou, nas Sessões 3 e 4, um desempenho similar ao apresentado pelos participantes da Condição RD.
Os resultados, mostrando que todos os participantes que apresentaram um desempenho dependente das conseqüências programadas na Sessão 3, deixaram de seguir a regra discrepante das contingências na Sessão 4 (caso dos Participantes P11, P12, P13, P14 e P25); e todos os que apresentaram um desempenho independente das conseqüências programadas na Sessão 3, seguiram a regra discrepante das contingências na Sessão 4 (caso dos Participantes P21, P22, P23 e P24); são similares aos resultados obtidos no estudo de Albuquerque e Silva (2006) e indicam que o seguimento de regras discrepantes das contingências pode depender das fontes de controle do comportamento alternativo ao especificado por essa regra na história experimental do ouvinte. Em outras palavras, esses resultados apóiam a proposição desses autores de que o seguimento de regras discrepantes das contingências: 1) tende a deixar de ocorrer quando, antes da apresentação da regra, o comportamento alternativo ao especificado pela regra discrepante mostra-se sensível às mudanças nas contingências (caso dos Participantes P11, P12, P13, P14 e P25); e 2) tende a ser 
mantido quando, antes da apresentação da regra, esse comportamento alternativo mostra-se insensível às mudanças nas contingências (caso dos Participantes P21, P22, P23 e P24).

Nessa linha de pesquisa, que investiga o controle por regras, alguns autores têm sugerido que se pode alterar a probabilidade futura do comportamento de seguir regras vir a ocorrer, manipulando-se as conseqüências produzidas por esse comportamento (Baron \& Galizio, 1983; Galizio, 1979; Monteles, Paracampo \& Albuquerque, 2006; Paracampo \& Albuquerque, 2004). Em adição, os resultados do presente estudo apóiam a sugestão de que isso também pode ser feito manipulando a história do ouvinte (Albuquerque e cols., 2006; Albuquerque \& Reis, 2007). Portanto, tal como esses estudos, os resultados do presente estudo também têm implicações práticas importantes, na medida em que sugerem procedimentos alternativos por meio dos quais se pode tornar o seguimento de regras discrepantes das contingências mais ou menos provável de ocorrer.

Se a manutenção do seguimento de regras discrepantes depende da história de sensibilidade às contingências do comportamento alternativo ao especificado pela regra discrepante, como se poderia tornar esse comportamento alternativo mais ou menos sensível às contingências? Tem sido sugerido que a forma por meio da qual um determinado comportamento é estabelecido, se por regras ou por modelagem ou por reforço diferencial, é um dos importantes fatores na determinação de sua sensibilidade às contingências. Tanto que tem sido sugerido que o comportamento tem maior probabilidade de mudar acompanhando mudanças nas contingências quando, antes dessa mudança, ele é estabelecido por modelagem ou reforço diferencial do que quando é estabelecido por regra correspondente (Paracampo e cols., 2001; Shimoff e cols., 1981). Os resultados do presente estudo apóiam essa sugestão, uma vez que todos os quatro participantes que tiveram o comportamento estabelecido por reforço diferencial na Sessão 2, mudaram os seus desempenhos acompanhando a mudança nas contingências na Sessão 3 (caso dos Participantes P11, P12, P13 e P14 da Condição RD); e quatro dos cinco participantes que tiveram o comportamento estabelecido por regra correspondente na Sessão 2, continuaram seguindo a regra na Sessão 3, isto é, não mudaram os seus desempenhos acompanhando a mudança nas contingências nesta sessão (caso dos Participantes P21, P22, P23 e P24 da Condição IN).
A análise, feita até momento, sugere que, quando o comportamento é estabelecido por regras, ele tende a se mostrar insensível à mudança nas contingências. Essa história de insensibilidade, por sua vez, tende a interferir no seguimento subseqüente de regras discrepantes, contribuindo para que esse comportamento seja mantido. Em contraste, quando o comportamento é estabelecido por contingências, ele tende a se mostrar sensível à mudança nas contingências. Essa história de sensibilidade, por sua vez, tende a interferir no seguimento subseqüente de regras discrepantes, contribuindo para que esse comportamento deixe de ocorrer.

No entanto, ainda faltar tentar esclarecer algumas diferenças entre os resultados aqui obtidos e os resultados encontrados em outros estudos. Comparando, então, os resultados observados na transição da Sessão 2 para a Sessão 3 do presente estudo com alguns resultados do estudo de Albuquerque e Silva (2006), constata-se que quatro dos cinco participantes (correspondente a $80 \%$ ), que tiveram o comportamento estabelecido por regra correspondente no presente estudo, mantiveram-se seguindo a regra após a mudança nas contingências, enquanto que apenas dois dos cinco participantes (correspondente a $40 \%$ ), que tiveram o comportamento estabelecido por regra correspondente no estudo de Albuquerque e Silva, fizeram o mesmo.

Os resultados do presente estudo, quando considerados isoladamente, não podem explicar essas diferenças. Contudo, quando o presente estudo é comparado com o de Albuquerque e Silva (2006), pode-se inferir que aquelas diferenças de resultados podem ter ocorrido, possivelmente, devido à combinação dos seguintes fatores: 1 ) a tarefa dos participantes era mais complexa no estudo de Albuquerque e Silva comparado com o presente estudo; 2) no estudo de Albuquerque e Silva o comportamento verbal que descrevia o nãoverbal foi solicitado aos participantes ao longo das sessões experimentais, enquanto no presente experimento esse comportamento não foi solicitado; e, 3) no estudo de Albuquerque e Silva o comportamento era reforçado em esquema CRF, antes e depois da mudança nas contingências, enquanto no presente experimento, o comportamento era reforçado em esquema FR 2, entes da mudança, e em esquema CRF, depois da mudança. Em outras palavras, quando os resultados desses dois estudos são analisados juntos, é possível especular que a maioria dos participantes do estudo Albuquerque e Silva abandonou o seguimento da regra após a mudança nas contingências, porque, em relação ao presente estudo, os participantes no estudo de Albuquerque e Silva foram expostos a número 
maior de fatores que, supostamente, teriam facilitado o controle pelas conseqüências programadas. Pode-se deduzir, então, que a maioria dos participantes do presente estudo persistiu seguimento a regra após a mudança nas contingências, supostamente, porque, em relação ao estudo Albuquerque e Silva, os participantes no presente estudo foram expostos a fatores que favoreciam o seguimento de regras.

Essa análise sugere que a persistência do seguimento de regras, após a mudança nas contingências, depende de fatores que facilitem a manutenção deste comportamento. Essa análise é, portanto, consistente com a visão de que o seguimento de regras é determinado por múltiplos fatores (Albuquerque e cols., 2003; Cerutti, 1989; Chase \& Danforth, 1991; Hayes e cols., 1986; Newman, Buffington \& Hemmes, 1995; Otto e cols., 1999), e com algumas evidências experimentais que têm mostrado que o seguimento de regras nem sempre persiste, quando as contingências são alteradas (Bernstein, 1988; Galizio, 1979; LeFrancois e cols., 1988; Michael \& Bernstein, 1991; Newman e cols. 1995; Paracampo \& Albuquerque, 2004; Santos, Paracampo \& Albuquerque, 2004; ver também Paracampo \& Albuquerque, 2005, para uma revisão).

O presente estudo, no entanto, não deixa claro de que maneira, qual daqueles três fatores poderia ter contribuído, ou não, para a ocorrência daquelas diferenças de resultados. Também não deixa claro porque aqueles três fatores, aparentemente, não interferiram na sensibilidade à mudança nas contingências no caso dos participantes que tiveram o comportamento estabelecido por reforço diferencial. Isto considerando que, tanto no presente estudo quanto no estudo anterior, esses participantes mudaram os seus desempenhos quando as contingências mudaram. Pesquisas futuras, utilizando o presente estudo como parâmetro, poderiam contribuir para fazer tais esclarecimentos. Tais investigações são importantes porque, embora haja algumas proposições sugerindo que as verbalizações dos participantes podem facilitar a discriminação das contingências (Cabello, Luciano, Gomez \& Barnes-Holmes, 2004; Dixon \& Hayes, 1998; Rosenfarb, Newland, Brannon \& Howey, 1992; Vaughan, 1985) e que é improvável que o seguimento de regras seja mantido quando o esquema programado para reforçar o nãoseguimento de regras é um esquema de reforço contínuo (Newman e cols., 1995), também há evidências experimentais mostrando que o seguimento de regras pode ser mantido, mesmo quando os participantes são solicitados, ao longo do experimento, a descrever o comportamento que produz reforço, e mesmo quando o esquema programado para reforçar o seguimento de regra, antes da mudança nas contingências, e o nãoseguimento de regra, depois da mudança nas contingências, é um esquema CRF (Paracampo e cols., 2001). Tais investigações também são importantes porque poucos estudos têm procurado avaliar o papel da complexidade da tarefa na sensibilidade do comportamento a mudanças nas contingências, embora haja evidências experimentais mostrando que a complexidade da tarefa pode interferir na ocorrência do seguimento de regras (Albuquerque \& Ferreira, 2001).

Passando, agora, a comparar os resultados observados na transição da Sessão 3 para a Sessão 4 do presente estudo com alguns resultados dos estudos da linha de pesquisa que tem investigado os efeitos de histórias comportamentais (Freeman \& Lattal, 1992; Wanchisen, Tatham \& Mooney, 1989; Weiner, 1964, 1969; ver também Tatham \& Wanchisen, 1998, e Weiner, 1983, para uma revisão), pode-se dizer que, de modo geral, os resultados do presente estudo são semelhantes a alguns resultados encontrados nessa outra linha de pesquisa. Por exemplo, os resultados do presente estudo, mostrando que alguns participantes seguiram a regra discrepante, enquanto outros deixaram de seguir esta regra, dependendo de suas histórias experimentais de sensibilidade à mudança nas contingências, são semelhantes aos resultados do estudo de Weiner (1969), que observou que humanos adultos responderam diferencialmente em esquema de intervalo fixo (FI), dependendo de suas histórias experimentais. Ou seja, os participantes com treino prévio em esquema de razão fixa responderam com taxas altas e constantes em FI. Diferentemente, os participantes com treino prévio em esquema de reforço diferencial de taxas baixas (DRL), responderam com taxas baixas em FI.

Mas uma análise mais minuciosa destes estudos pode revelar que os resultados do estudo de Weiner (1969) se assemelham mais aos resultados obtidos na Condição RD (Reforço Diferencial) do que aos obtidos na Condição IN (Instrução) do presente estudo. Por exemplo, os procedimentos geralmente usados nos estudos planejados mais especificamente para avaliar os efeitos de histórias comportamentais sobre a emissão subseqüente de comportamentos em esquemas de reforço, consistem, em geral, em uma fase de treinamento (na qual um comportamento - não estabelecido por regra - é mantido em um esquema de reforço) e em uma fase de teste (na qual os efeitos da história, construída na primeira fase, são avaliados em 
um outro esquema de reforço). Sugere-se que os efeitos de histórias comportamentais ocorrem quando, na fase de teste, o participante continua apresentando (ou volta a apresentar) o desempenho que vinha sendo reforçado na fase de treino (Wanchisen, 1990). Similarmente, na Condição RD do presente estudo, também se pode dizer que a história comportamental exerceu controle porque o comportamento previamente reforçado na Sessão 3 voltou a ser emitido na Sessão 4, quando o seguimento da regra discrepante deixou de ocorrer. Ou seja, neste caso, o controle pela história experimental substituiu o controle pela regra. Contudo, não se pode dizer o mesmo em relação aos dados da Condição IN do presente estudo, porque, nesta condição, o comportamento observado na Sessão 3 não continuou sendo emitido na Sessão 4. Nesta condição, os comportamentos apresentados nas Sessões 3 e 4 foram comportamentos diferentes, com topografias diferentes e sob controle de variáveis diferentes. Ou seja, na Sessão 3, a seqüência CEF foi emitida, possivelmente, sob controle da história de reforço para o seguimento da regra correspondente, construída na Sessão 2. Já na Sessão 4, a seqüência FCE foi emitida sob controle da regra discrepante, possivelmente, devido à história de insensibilidade observada na Sessão 3. Assim, enquanto a história da Condição RD contribuiu para que o seguimento da regra discrepante fosse abandonado, a história da Condição IN contribuiu para que esse comportamento fosse mantido. Pesquisas futuras poderiam esclarecer os mecanismos pelos quais as histórias de seguimento de regras exercem seus efeitos.

\section{REFERÊNCIAS}

Albuquerque, L. C. (1991). Efeitos de regras no controle do comportamento humano [Resumo]. Em Sociedade Brasileira de Psicologia (Org.), Resumos de comunicações científicas (p. 162). Ribeirão Preto: SBP.

Albuquerque, L. C. (2001). Definições de regras. Em H. J. Guilhardi, M. B. B. P. Madi, P. P. Queiroz \& M. C. Scoz (Orgs.), Sobre comportamento e cognição: Expondo a variabilidade (p. 132-140). Santo André: ARBytes.

Albuquerque, L. C., \& Ferreira, K. V. D. (2001). Efeitos de regras com diferentes extensões sobre o comportamento humano. Psicologia: Reflexão e Crítica, 14, 143-155.

Albuquerque, L. C., \& Reis, A. A. (2007). Efeitos de histórias de reforço, curtas e prolongadas, sobre o seguimento de regras. Manuscrito submetido à publicação.

Albuquerque, L. C., Reis, A. A., \& Paracampo, C. C. P. (2006). Efeitos de uma história de reforço contínuo sobre o seguimento de regra. Acta Comportamentalia, 14, 47-75.

Albuquerque, L. C., Santos, A. C. S., Silva, F. M., Mendonça, P. S. M., Queiroz, T. M. M., \& Silva, W. C. M. P. (1993). Análise do papel das conseqüências programadas no seguimento de regras [Resumo]. Em Sociedade Brasileira para o Progresso da Ciência (Org.), Anais da 45 Reunião Anual da Sociedade Brasileira para o Progresso da Ciência (p. 903). Recife: SBPC.

Albuquerque, L. C., \& Silva, F. M. (2006). Efeitos da exposição a mudanças nas contingências sobre o seguir regras. Psicologia: Teoria e Pesquisa, 22, 101-112.

Albuquerque, L. C., de Souza, D. G., Matos, M. A., \& Paracampo, C. C. P. (2003). Análise dos efeitos de histórias experimentais sobre o seguimento subseqüente de regras. Acta Comportamentalia, 11, 87-126.

Baron, A., \& Galizio, M. (1983). Instructional control of human operant behavior. The Psychological Record, 33, 495-520.

Bernstein, D. J. (1988). Laboratory lore and research practices in the experimental analysis of human behavior: Designing session logistics-how long, how often, how many? The Behavior Analyst, 11, 51-58.

Branch, M. N. (1991). On the difficulty of studying "basic" behavioral processes in humans. The Behavior Analyst, 14, 107-110.

Cabello, F., Luciano, C., Gomez, I., \& Barnes-Holmes, D. (2004). Human schedule performance, protocol analysis, and the "silent dog” methodology. The Psychological Record, 54, 405422.

Cerutti, D. T. (1989). Discrimination theory of rule-governed behavior. Journal of the Experimental Analysis of Behavior, 51, 259-276.

Cerutti, D. T. (1991). Discriminative versus reinforcing properties of schedules as determinants of schedule insensitivity in humans. The Psychological Record, 41, 51-67.

Chase, P. N., \& Danforth, J. S. (1991). The role of rules in concept learning. Em L. J. Hayes \& P. N. Chase (Orgs.), Dialogues on verbal behavior (p. 205-225). Hillsdale, NJ: Erlbaum.

Dixon, M. R., \& Hayes, L. J. (1998). Effects of differing instructional histories on the resurgence of rule-following. The Psychological Record, 48, 275-292.

Freeman, T. J., \& Lattal., K. A. (1992). Stimulus control of behavioral history. Journal of the Experimental Analysis of Behavior, 57, 5-15.

Galízio, M. (1979). Contincency-shaped and rule-governed behavior: Instructional control of human loss avoidance. Journal of Experimental Analysis of Behavior, 31, 53-70.

Hayes, S. C., Brownstein, A. J., Zettle, R. D., Rosenfarb, I., \& Korn, Z. (1986). Rule governed behavior and sensitivity to changing consequences of responding. Journal of the Experimental Analysis of Behavior, 45, 237-257.

Joyce, J. H., \& Chase, P. N. (1990). Effects of response variability on the sensivity of rule-governed behavior. Journal of the Experimental Analysis of Behavior, 54, 251-262.

LeFrancois, J. R., Chase, P. N., \& Joyce, J. (1988). The effects of variety of instructions on human fixed-interval performance. Journal of the Experimental Analysis of Behavior, 49, 383-393.

Lowe, C. F. (1979). Determinants of human operant behaviour. Em M. D. Zeiler \& P. Harzem (Orgs.), Advances in analysis of behaviour: Vol. 1 - Reinforcement and the organization of behaviour (pp. 159-192). Chichester, England: Wiley. 
Martinez, H., \& Tomayo, R. (2005). Interactions of contingencies, instructional accuracy, and instructional history in conditional discrimination. The Psychological Record, 55, 633-646.

Michael, R. L., \& Bernstein, D. J. (1991). Transient effects of acquisition history on generalization in a matching-to-sample task. Journal of the Experimental Analysis of Behavior, 56, 155-166.

Monteles, K. M. C., Paracampo, C. C. P., \& Albuquerque, L. C. (2006). Efeitos de uma história de reforço contínuo e de conseqüências sociais sobre o seguir regras. Psicologia: Reflexão $e$ Crítica, 19, 186-196.

Newman, B., Buffington, D. M., \& Hemmes, N. S. (1995). The effects of schedules of reinforcement on instruction following. The Psychological Record, 45, 463-476.

Otto, T. L., Torgrud, L. J., \& Holborn, S. W. (1999). An operant blocking interpretation of instructed insensitivity to schedule contingencies. The Psychological Record, 49, 663-684.

Paracampo, C. C. P., \& Albuquerque, L. C. (2004). Análise do papel das conseqüências programadas no seguimento de regras. Interação em Psicologia, 8, 237-245.

Paracampo, C. C. P., \& Albuquerque, L. C. (2005). Comportamento controlado por regras: Revisão crítica de proposições conceituais e resultados experimentais. Interação em Psicologia, 9, 227-237.

Paracampo, C. C. P., de Souza, D. G., Matos, M. A., \& Albuquerque, L. C. (2001). Efeitos de mudanças em contingências de reforço sobre o comportamento verbal e o não verbal. Acta Comportamentalia. 9, 31-55.

Rosenfarb, I. S., Newland, M. C., Brannon, S. E., \& Howey, D. S. (1992). Effects of self-generated rules on the development of schedule-controlled behavior. Journal of the Experimental Analysis of Behavior, 58, 107-121.

Santos, J. G. W., Paracampo, C. C. P., \& Albuquerque, L. C. (2004). Análise dos efeitos de histórias de variação comportamental sobre o seguimento de regras. Psicologia: Reflexão e Crítica, 17, 413-425.

Schlinger, H. (1993). Separating discriminative and functionaltering effects of verbal stimuli. The Behavior Analyst, 16, 923.

Schlinger, H., \& Blakely, E. (1994). A descriptive taxonomy of environmental operations and its implications for behavior analysis. The Behavior Analyst, 17, 43-57.
Shimoff, E., Catania, A. C., \& Matthews B. A. (1981). Uninstructed human responding: Sensitivity of low-rate performance to schedule contingencies. Journal of the Experimental Analysis of Behavior, 36, 207-220.

Skinner, B. F. (1969). Contingencies of reinforcement: A theoretical analysis. New York: Appleton-Century-Crofts.

Skinner, B. F. (1974). About behaviorism. New York: Alfred A. Knopf.

Vaughan, M. E. (1985). Repeated acquisition in the analysis of rule-governed behavior. Journal of the Experimental Analysis of Behavior, 44, 175-184.

Tatham, T. A., \& Wanchisen, B. A. (1998). Behavioral history: A definition and some common findings from two areas of research. The Behavior Analyst, 21, 241-251.

Torgrud, L. J., \& Holborn, S. W. (1990). The effects of verbal performance descriptions on nonverbal operant responding. Journal of the Experimental Analysis of Behavior, 54, 273-291.

Vaughan, M. E. (1985). Repeated acquisition in the analysis of rule-governed behavior. Journal of the Experimental Analysis of Behavior, 44, 175-184.

Wanchisen, B. A. (1990). Forgetting the lessons of history. The Behavior Analyst, 13, 31-37.

Wanchisen, B. A., Tatham, T. A., \& Mooney, S. E. (1989). Variable-ratio conditioning history produces high- and low-rate fixed-interval performance in rats. Journal of the Experimental Analysis of Behavior, 52, 167-179.

Weiner, H. (1964). Conditioning history and human fixed-interval performance. Journal of the Experimental Analysis of Behavior, 7, 383-385.

Weiner, H. (1969). Controlling human fixed-interval performance. Journal of the Experimental Analysis of Behavior, 12, 349-373.

Weiner, H. (1983). Some thoughts on discrepant human-animal performances under schedules of reinforcement. The Psychological Record, 33, 521-532.

\section{Notas:}

1 Este trabalho foi baseado nos dados da dissertação de mestrado do primeiro autor apresentada em 2003 no Programa de PósGraduação em Teoria e Pesquisa do Comportamento/UFPA.

2 Regras são estímulos antecedentes verbais que podem especificar contingências (Skinner, 1969, 1974) e exercer múltiplas funções, isto é, regras podem tanto evocar comportamento quanto alterar as funções dos estímulos por elas descritos (Albuquerque, 1991, 2001; ver também Schlinger, 1993, 1994, para uma análise dessas funções do ambiente). Instruções, conselhos, ordens, etc, são exemplos particulares de regras. No presente estudo o termo "regra" está sendo usado em situações mais amplas e o termo "instrução” está sendo usado em situações mais restritas, como nas descrições dos procedimentos e resultados de pesquisas. 
3 No presente estudo está sendo usado o termo sensibilidade para descrever o comportamento que está sob controle de suas conseqüências imediatas e o termo insensibilidade para descrever o comportamento que não está sob controle de suas conseqüências imediatas em uma determinada situação particular (Albuquerque e cols., 2003). Por essa definição, um critério para avaliar a sensibilidade ou a insensibilidade do comportamento é verificar se ele ocorre sob controle de suas conseqüências imediatas ou se ocorre independentemente de tais conseqüências. Isso pode ser feito, tanto quando se utiliza o procedimento tradicional, quanto quando se utiliza o procedimento alternativo, e não apenas quando se utiliza o procedimento tradicional, como outras definições destes termos parecem sugerir (Albuquerque \& Reis, 2007).

4 Para evitar coincidência entre o comportamento descrito pela instrução correspondente no início da segunda sessão da Condição Instrução e o comportamento apresentado pelo participante no início da primeira sessão dessa condição, esta instrução podia especificar a seqüência alternativa ECF, descrita entre colchetes.

5 A Figura 1 não apresenta os dados da Sessão 1, porque nessa sessão as seqüências não foram classificadas de corretas e incorretas, uma vez que não eram reforçadas.

\section{Sobre os autores:}

Laercio de Sousa Silva: Psicólogo e Mestre em Teoria e Pesquisa do Comportamento pela Universidade Federal do Pará.

Luiz Carlos de Albuquerque: Professor da Universidade Federal do Pará e bolsista de produtividade em pesquisa do CNPq.

Endereço para correspondência: Luiz Carlos de Albuquerque - Rua Oliveira Belo, 238/1702 - Umarizal - Belém/PA - CEP 66050380. Endereço eletrônico: lcalbu@ufpa.br 